\title{
The Influence of Functional Carrier Particles (FCPs) on the Molecular Transport Rate Through the Reconstructed Bronchial Mucus: In Vitro Studies
}

\author{
Marcin Odziomek • Tomasz R. Sosnowski • \\ Leon Gradoń
}

Received: 27 February 2014 / Accepted: 15 October 2014 / Published online: 8 November 2014

(C) The Author(s) 2014. This article is published with open access at Springerlink.com

\begin{abstract}
Mass transfer of deposited drug particles in the human lungs is an important factor in the effective drug delivery by inhalation. This study is focused on the basic research related to the possible acceleration of drug penetration toward the intended site of action through the barrier created by mucus layer. It is proposed that beneficial effect can be obtained by altering the mucus structure by inhaled functional carrier particles (FCPs) which are used to enhance pulmonary delivery of aerosolized powder drugs and simultaneously contain mucolytics (e.g., $N$-acetylcysteine: NAC). The FCPs were prepared by an optimized spray-drying technique and tested regarding their influence on the rheology of the reconstructed bronchial mucus and the transport rate of model drugs (Rhodamine B and Disodium Cromoglycate). The results indicate that the viscosity of mucus with high mucin concentration (i.e., reflecting a disease is reduced by up to $42 \%$ depending on the concentration of added FCPs what is similar to the effect of pure NAC. Simultaneously, the effective diffusion coefficient $D_{\text {eff }}$ of the model drug through the mucus increased from $23 \times 10^{-7}$ (pure mucus) to $42 \times 10^{-7} \mathrm{~cm}^{2} / \mathrm{s}$ (mucus modified by FCPs), and it was comparable to the effect obtained with pure NAC $\left(51 \times 10^{-7} \mathrm{~cm}^{2} / \mathrm{s}\right)$. The results confirm that using mucolytics incorporated into powder particles (drug carriers) may be used to enhance mass transfer of inhaled aerosol drugs across the bronchial mucus layer.
\end{abstract}

Keywords Artificial mucus · Viscosity · Mass transport · Drug carriers · Mucolytics

\footnotetext{
M. Odziomek $(\varangle) \cdot$ T. R. Sosnowski · L. Gradoń

Faculty of Chemical and Process Engineering, Warsaw University of Technology,

1 Waryńskiego Street, 00-645 Warsaw, Poland

e-mail: M.Odziomek@ichip.pw.edu.pl

T. R. Sosnowski

e-mail: T.Sosnowski@ichip.pw.edu.pl

L. Gradoń

e-mail: L.Gradon@ichip.pw.edu.pl
} 


\section{Introduction}

Inhalation of aerosolized drugs is the most efficient method of therapy of the respiratory tract diseases, regardless of two natural mechanisms that play key role in protection of the human respiratory tract against negative influence of pathogens and pollutants present in inhaled air (Gradoń and Marijnissen 2003). One of these mechanisms is related to airway tree structure which causes that majority of inhaled particles (including drugs) cannot penetrate to the lower parts of the lungs. Only particles with clearly defined aerodynamic diameter (below $5 \mu \mathrm{m}$ ) can overcome this barrier and deposit in the tracheobronchial tree with relatively high efficiency. However, such as any other deposited strange matter, they are removed from the surface of the respiratory tract by second defense mechanism, called mucociliary clearance, which transports particles to the upper airways. The effective aerosol therapy requires that bath barriers are overcome.

In this paper, we focus on the role of the functional carrier particles (FCPs) which have been proposed as novel excipients in dry powder inhalers (DPIs) which may help to achieve this goal and ensure more efficient inhalation therapy (Odziomek et al. 2012). Briefly summarizing this concept, it is based on the fact that viscous properties of tracheobronchial mucus significantly affect the rate of absorption and action of drugs administered by the pulmonary route (Varum et al. 2010), so the natural way to improve this situation is simultaneous delivery of drug and mucoactive agent in the form of inhalation of rationally designed powder. Changes in mucus composition which are typically associated to diseased conditions, result in modification of mucus crosslink density and-as a consequence-in its rheological properties. In asthma, COPD or cystic fibrosis mucus viscoelasticity is increased by several times what significantly disturbs the function of "mucociliary escalator." Thickness of mucus layer becomes much higher than normally (up to 100-200 $\mu \mathrm{m}$ ), so the effectiveness of transmucosal penetration of inhaled drugs is significantly reduced (Cone 2009; McGill and Smyth 2010).

The main components of mucus with the biggest influence on its rheological properties (except water) and crosslink density are glycoproteins known as mucins. Mucins are built up of subunits that are bound to each other by covalent bonds (disulfide bridges) between cysteine residues. In addition to mucins, a large number of other constituents are present in the mucus at lower concentrations, such as proteins, lipids, cellular and serum macromolecules, DNA, and electrolytes (Sanders et al. 2000). The gel-like behavior of mucus arises mainly from physical chain entanglements and intermolecular interactions between mucins. However, mucins can also interact with DNA, proteins, and lipids (Bansil and Turner 2006). All these interactions (covalent bonds, ionic bonds, hydrogen bonds, and van der Waals forces) enhance the viscoelasticity of mucus and establish a network structure (King 2005). As empirical results indicate the average pore size of the tracheobronchial mucus in cystic fibrosis is in the range of 100-200 nm (Matsui et al. 2006). It is clear that transport of particles or molecules with higher diameter than mucus mesh pore size will be retarded. However, also transport of smaller molecules is inhibited in polymer solutions like mucus. Partly, it can be explained by electrostatic or hydrophobic interactions between diffusing molecules and mucus compounds (Bhat et al. 1995; Meaney and O'Driscoll 1999; Mortazawi 1995). However, it is not sufficiently comprehensive explanation of these phenomena. The polymer may also influence solute diffusion in other way. The presence of impenetrable, slow moving polymer molecules in a solution may lead to an increase in the path length for diffusion. Solute movement may be also retarded by increased hydrodynamic drag. In general, the diffusivity of a solute through physically cross-linked polymer solution decreases as cross-linking density increases. This phenomenon was discussed in many papers and can be 
Fig. 1 Concept of FCP activity

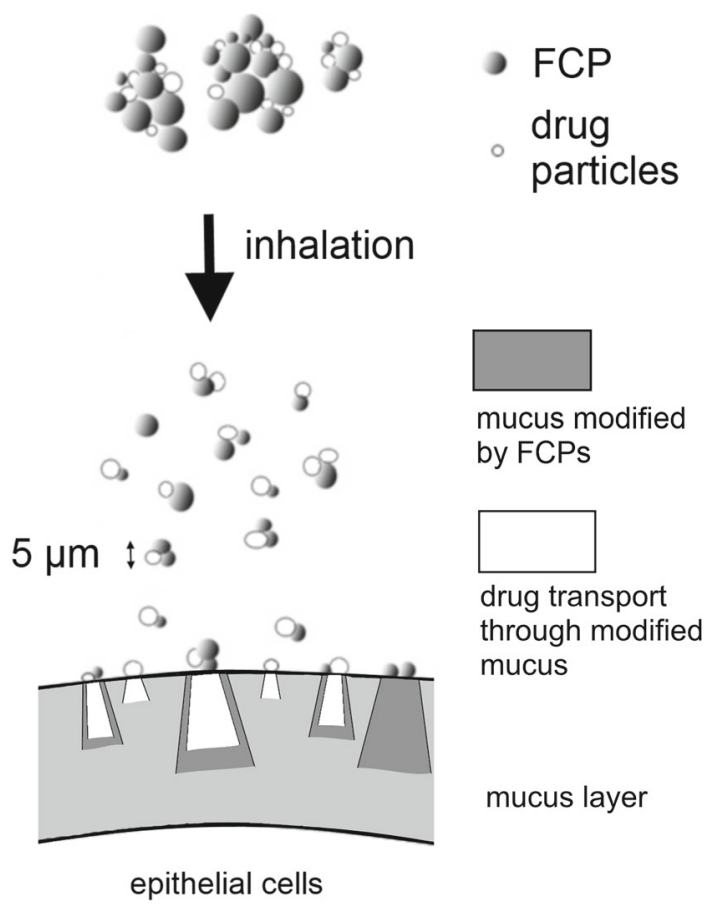

described, by many models, e.g., free volume theory (Flynn et al. 1974; Muhr and Blanshard 1982; Wesselingh 1993). As observed, reduction of length of polymer chains takes effect in a faster transport of solutes through the mucus (Gao and Fagerness 1995). It was confirmed by clinical trials. In some cases, improvement in the efficacy of treatment of respiratory tract diseases (e.g., asthma, COPD, and cystic fibrosis) may be achieved by direct administration of the mucolytic agents, such as $N$-acetylcysteine (NAC), dithiothreitol, bromhexine, or erdosteine, prior to inhalation of the dedicated drug. Mucolytics delivered typically as liquid aerosol (droplets made of mucolytic solution) modify the mucus structure by splitting glycoproteins into smaller subunits by reduction of disulfur bonds. Consequently, the mucus loses its viscoelastic properties and becomes more 'transparent' for drug molecules penetration (Ferrari et al. 2001; Stern et al. 1998).

The proposed concept of FCPs allows avoiding the need of the sequential treatment, since mucolytics are incorporated directly into solid particles required as drug carrier in powder formulations. According to this idea, the aggregates which are formed of FCPs and drugs (as submicron or nanostructured particles), will deposit together (i.e. at the same site) on the surface of the mucus layer in the respiratory tract, Fig. 1. In most cases, the dissolution of amorphous powders does not limit the drug transport through mucus (Patton et al. 2010). After fast powder dissolution, slow diffusion of a drug through the mucus layer starts. Simultaneously, the mucolytic agent released from FCPs begins to modify the mucus structure and facilitates the transport of the co-released drug molecules to the epithelial cells * receptors. In this way, the local mucolytic activity induced by one component improves the penetration of the other component (drug) to the epithelial cells what may be favorable especially in delivering of quick-relief and short-acting drugs. However, FCPs may also facilitate transport of nanostructural particles to the inner mucus layer (sol layer) which is 
removed from tracheobronchial tree slower than the outer one (gel layer). Nanostructural particles trapped in this layer can release drugs molecules for prolonged time, what may minimize frequency of the required inhalations during a day (Almeida and Souto 2007; Fischer et al. 2009; Lai et al. 2007; Wang et al. 2008; Yoncheva et al. 2005).

In the previous work (Odziomek et al. 2012), we have shown that FCPs can be conveniently produced by spray drying in controlled conditions using appropriately selected aqueous precursors containing compounds currently approved for pharmaceutical use, such as $\mathrm{N}$ acetylcystine, or which are waiting for the approval-like low molecular-weight dextran (LMWD). Dextran was used as the stabilizer of prepared powder particles because pure NAC forms hygroscopic crystals which are unsuitable for inhalation. Incorporation of the LMWD allows delivering mucoactive particles to the tracheobronchial tree in the form of aerosol released from a dry powder inhaler (DPI). In addition, LMWD is a convenient adjuvant in this case since it shows mucolytic properties itself and may also improve the mucociliary clearance. According to literature data, dextran acts probably by an osmotic mechanism (attraction of fluid into the airway milieu) and additionally by disruption of the network crosslinks between neighboring mucin macromolecules (Feng et al. 1997; Hirji et al. 1998; King and Rubin 2002; Sudo et al. 2000).

We used mixtures of LMWD and NAC in proportion-4:1 and 1:1. The optimum conditions of the spray drying process for obtaining FCPs (drying air temperature: $120^{\circ} \mathrm{C}$, solution feed: $3 \mathrm{ml} / \mathrm{min}$, aspirator rate: $37 \mathrm{~m}^{3} / \mathrm{h}$, airflow: $0.475 \mathrm{~m}^{3} / \mathrm{h}$, and outflow temperature: $55^{\circ} \mathrm{C}$ ) allowed to maximize powder yield and the content of particles smaller than $5 \mu \mathrm{m}$ (fine particle fraction-FPF), i.e., particles expected to penetrate and deposit in the tracheobronchial tree with the highest efficiency (Heyder 2004; Koushik and Kompella 2004). Both, FPF and mass median aerodynamic diameter (MMAD) obtained after dispersion of FCPs from a commercial capsule-type DPI (FPF: 46-51\%, MMAD: 2.28-2.43 $\mu \mathrm{m}$ ) indicate that these aerosols should have high deposition in the respiratory tract and, in consequence, they are applicable in pulmonary drug delivery. Moreover, the relatively high glass transition temperature $\mathrm{Tg}\left(51-61^{\circ} \mathrm{C}\right)$ of the composite material of FCPs should minimize the tendency to recrystallization which is typical for amorphous solids. In consequence, it should ensure stability of their most important parameters during storage (Odziomek et al. 2012).

However, as it was noted earlier, effective deposition in the target area in the lungs is only the first step of drug delivery-the second one is its transport through the mucus. Although it is well known that NAC delivered to the lungs as aerozolized aqueous solution decreases mucus viscosity in the airways, there is still no information about the influence of NAC delivered as powder on mucus rheology (viscosity) and on the mass transfer in the mucus layer. Therefore, this study is focused on determination how each chemical compound present in the FCP influences mucus properties and, finally, if a synergic effect of all components may be obtained using FCPs. We limit this research only to FCPs characterized with the best aerodynamic performance and stability (Odziomek et al. 2012) and two types of mucin solutions used to reflect properties of natural mucus in different physiological states.

\section{Materials and Methods}

\subsection{Materials}

$N$-acetylcysteine (NAC, A7250), low molecular-weight dextran (LMWD, 31388), and mucin (type II, M2378) were purchased from Sigma-Aldrich (USA). Rhodamine B, phosphate 
Table 1 Content of functional carrier particles and their components in mucus samples with both considered mucin concentrations

\begin{tabular}{|c|c|c|c|c|c|c|c|}
\hline \multirow{2}{*}{$\begin{array}{l}\text { Components added to mucus } \\
\text { NAC }\end{array}$} & \multicolumn{7}{|c|}{ Content in the mucus $(\% \mathrm{w} / \mathrm{w})$} \\
\hline & 0 & $(0.25)$ & 0.5 & $(0.75)$ & 1 & 1.5 & 2.0 \\
\hline \multicolumn{8}{|l|}{ LMWD (Dextran $4000 \mathrm{Da})$} \\
\hline \multicolumn{8}{|l|}{ Functional Carrier NAC/Dextran 1:1 (FCP1) } \\
\hline Functional Carrier NAC/Dextran 1:4 (FCP2) & & & & & & & \\
\hline
\end{tabular}

Values in brackets concern samples with higher mucin concentration only $(20 \% \mathrm{w} / \mathrm{w})$

buffer, and sodium azide were purchased from POCH (Poland). Disodium Cromoglycate (DSCG) was donated by GSK Pharmaceuticals SA (Poland). Water used for preparing mucus solutions and for other analytical purposes were purified by reverse osmosis-RO (Puricom, USA).

\subsection{Preparation of Reconstructed Bronchial Mucus}

The artificial bronchial mucus model was reconstructed from the main component of natural mucus-mucin, according to the information from different literature sources (Bhat et al. 1996a, b; Dawson et al. 2004; McGill and Smyth 2010). According to manufacturer's data, the selected mucin was not modified by proteolic enzymes, so its natural structure should be preserved. Its final concentration after dissolution in the phosphate buffer $(7.4 \mathrm{pH})$ was adjusted to 10 and $20 \% \mathrm{w} / \mathrm{w}$. The $\mathrm{pH}$ value was precisely controlled and, if necessary, adjusted with $\mathrm{NaOH}$ aq. solution. A small amount $(0.02 \% \mathrm{w} / \mathrm{w})$ of sodium azide was added to protect the samples from microbial contamination. After setting the $\mathrm{pH}$ value and adding all components, mucus samples were gently stirred for 6 hours at a $37^{\circ} \mathrm{C}$. Preparation of different samples required addition of the strictly defined amounts of investigated compounds (data shown in Table 1). After the addition, $\mathrm{pH}$ was adjusted again, if necessary. Modified mucus samples were stirred with the same intensity during additional 2 hours at constant temperature $\left(37^{\circ} \mathrm{C}\right)$.

\subsection{Determination of the Apparent Mucus Viscosity}

Rheological data for prepared mucus samples were obtained with a rotational rheometer (Smart-Fungilab, Spain) equipped with two different spindles: LCP and TL5, designed for measurements in samples of significantly different viscosities (like samples with 10 and $20 \%$ w/w mucin concentration). For a straightforward comparison of the results, all measurements were done at the same rotational speed of a spindle $(6 \mathrm{rpm})$ and at similar shear rate $\dot{\gamma} \approx 8 \mathrm{~s}^{-1}$ which lies within the range found in bronchial mucus at normal physiological conditions $\left(0.1-20 \mathrm{~s}^{-1}\right)$ (Cone 2009). Since the mucus is a non- Newtonian fluid, we characterize its rheology by "apparent viscosity" understood as the viscosity measured at the physiological strain rate, which was applied in our studies. All viscosity measurements were taken at constant temperature $37^{\circ} \mathrm{C}$ (physiological conditions). In the first two series, the influence of each component of functional carrier particles (LMWD, NAC) was analyzed separately. In each series, the samples were studied during a successive increase of the concentration of added components $(0.25-2 \% \mathrm{w} / \mathrm{w})$. In the last two series of measurements, the influence of functional carrier particles (FCP1, FCP2) on mucus viscosity was investigated. The composition of tested mucus samples is shown in Table 1. 


\section{donor compartment acceptor compartment}

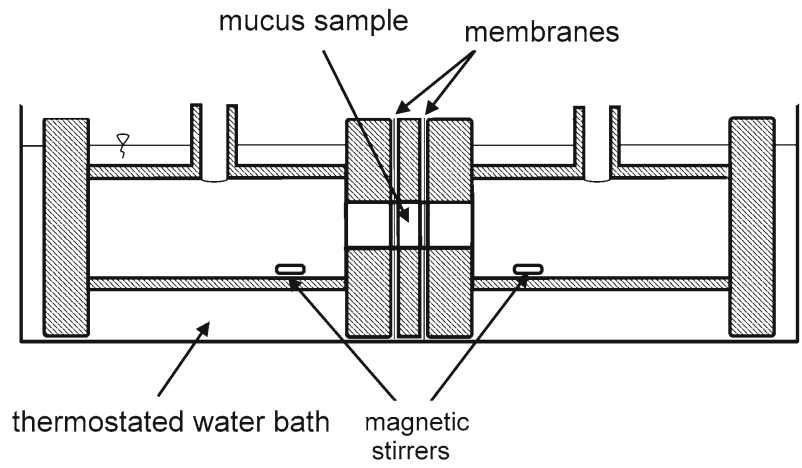

Fig. 2 Scheme of a typical diffusion chamber used to measure drug transport through the artificial mucus

\subsection{Mass Transfer Rate Through the Mucus Layer}

Mass transfer studies were done for Rhodamine B and disodium cromoglycate (DSCG) as model drugs. Their effective diffusion coefficient $D_{\text {eff }}$ in the mucus was measured using diffusion chamber method (Fig. 2).

The chamber has three compartments: a donor and a receiver (volume of each: $5 \mathrm{ml}$ ) separated by a thin layer of mucus solution restricted by two PVDF (polyvinyl difluoride) Durapore membranes (pore size $0.1 \mu \mathrm{m}$, thickness $0.125 \mathrm{~mm}$-Millipore, USA). The central holder had thickness of $3 \mathrm{~mm}$ and contained a fixed volume of mucus $(300 \mu \mathrm{l})$, which was injected with a syringe through a port on the top of this unit. The membranes were selected according to literature data (Norris and Sinko 1997) to avoid any leakage.

The donor compartment was filled with a solution containing either Rhodamine B (concentration of $0.05 \mathrm{mg} / \mathrm{ml}$ ) or DSCG (concentration of $0.1 \mathrm{mg} / \mathrm{ml}$. The receiver part was filled with the pure phosphate buffer ( $\mathrm{pH}$ 7.4). Solutions in both compartments were gently agitated by magnetic microstirrer $(300 \mathrm{rpm})$ to reduce the local mass transfer resistance near the membrane surface by hydrodynamic mechanism. The mucus layer was stagnant. A sample from receiver compartment sample was taken after approximately $5 \mathrm{~min}$ to check for any leaks. During whole experiment (6hours), samples for analysis were taken from the receiver at $30 \mathrm{~min}$ intervals. The content of Rhodamine B in was determined using fluorescence spectrometer (Lumina-Thermo Scientific, USA) at the excitation and emission wavelengths of 555 and $580 \mathrm{~nm}$, respectively. In case of DSCG, the concentration in samples was measured with HPLC system (model Infinity 1220, Agilent Technologies, USA) equipped with UV-Vis detector and $100 \mathrm{~mm}$ length ZORBAX Eclipse Plus C18. DSCG was analyzed at $325 \mathrm{~nm}$ wavelength. Compositions of mucus studied in this part of investigations are shown in Table 2.

The experiments were run in triplicate and their results were expressed as the mean \pm standard deviation.

The effective diffusion coefficient in the mucus layer, $D_{\text {eff }}$, was calculated from the appropriate solution of the Fick's law with the assumption of the steady-state process (Desai and Vadgama 1991). It was also assumed that membrane resistance is negligible and the mass transfer resistance at the membrane surface in both diffusion chambers is eliminated by stirring. Accordingly, the main resistance of mass transfer from donor phase to acceptor phase 
Table 2 Content of functional carrier particles and their components in mucus samples with mucin concentration $(20 \% \mathrm{w} / \mathrm{w})$

\begin{tabular}{lllll}
\hline Components added to mucus & \multicolumn{3}{l}{ Content of examined substances in the mucus $(\% \mathrm{w} / \mathrm{w})$} \\
\hline NAC & 0 & 0.5 & 1.0 & $(2.0)$ \\
Dextran (4000Da) & & & & \\
functional carrier NAC/Dextran 1:1 (FCP1) & & &
\end{tabular}

Value in bracket concern samples with $10 \% \mathrm{w} / \mathrm{w}$ mucin concentration

Fig. 3 Indications and concentration profile in diffusion chambers

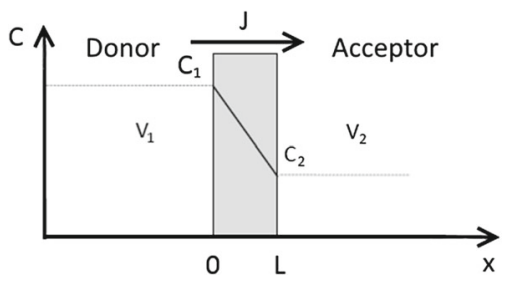

is located in the mucus layer of known thickness. For the assumed steady-state diffusion, the time necessary for solute transport through mucus, $t_{\mathrm{D} \text { mucus }}$, is significantly shorter than the time necessary to change its concentration in the diffusion cells, $t_{\mathrm{c}}$. This is correct only if the volume of mucus is significantly smaller than cells volume (Truskey et al. 2009), which is true for our system.

In this situation, mass flux of a solute transported from donor chamber to acceptor part can be expressed as

$$
J=-D_{\text {eff }} \frac{\mathrm{d} C}{\mathrm{~d} x}=D_{\text {eff }} \frac{\left(C_{1}-C_{2}\right)}{L}
$$

because the concentration distribution in the mucus layer is described by linear function (Fig. 3)

$$
C(x)=C_{1}+\left(C_{2}-C_{1}\right) \frac{x}{L}
$$

where $C_{1}$ - concentration of analyzed substance in donor compartment (and for $x=0$ ), $C_{2}$ concentration in the acceptor compartment (and for $x=L$ ), $x$-is the horizontal distance measured from the surface of the membrane in the donor compartment, and $L$-thickness of the mucus layer.

As the measurements were conducted in closed system, the concentration of analyzed substances in both diffusion chambers should change during the experiment and, for sufficiently long time, the concentration in both compartments will equalize. However, because of limited time of measurement (6hours), the solute concentration in the donor chamber was always at least two orders of magnitude higher than in the acceptor chamber. In each trial, the linear relationship of concentration against time was obtained $\left(R^{2}=0.95-0.99\right)$ what confirms that process was realized under the quasi-state conditions. Therefore, we can write

$$
\begin{aligned}
J=-D_{\text {eff }} \frac{\mathrm{d} C}{\mathrm{~d} x} & =D_{\text {eff }} \frac{\left(C_{1}-C_{2}\right)}{L}=\frac{1}{A} \frac{\mathrm{d} m}{\mathrm{~d} t} \\
D_{\text {eff }} & =\frac{L}{A\left(c_{1}-c_{2}\right)} \frac{\mathrm{d} m}{\mathrm{~d} t}
\end{aligned}
$$




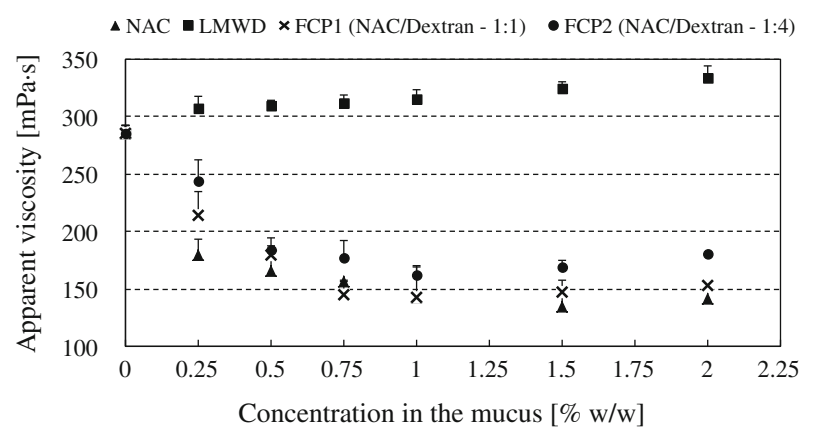

Fig. 4 Comparison of the apparent viscosity of the artificial bronchial mucus modified by functional carrier particles (FCPs) and their components. Average \pm SD, number of replicates $=3$

where $A$ - the surface area of mucus available for mass transfer, $\frac{d m}{d t}$ - the slope of the straight line in the graph of $m$ (total amount of solute transferred through the layer) versus $t$ (time) at steady state,

Described procedure of determination of the effective diffusion coefficient is similar to the presented by other authors (Brodin et al. 2010; Desai and Vadgama 1991; Shaw et al. 2004).

\section{Results and Discussion}

As the first step, it was checked if FCPs have ability to modify the structure of artificial mucus. This was determined by viscosity measurements of mucus samples (10 and 20\% w/w of mucins) after addition of strictly defined amounts of FCPs and their components (Table 1). The $\mathrm{pH}$ value of mucus samples was carefully controlled at physiological value (7.4) to ensure measurement accuracy and repeatability. Changes in $\mathrm{pH}$ could induce mucins gelation, which involves interactions of side chains of amino acids with pKs around 4, such as Asp or Glu from the non-glycosylated regions of the molecule. At neutral $\mathrm{pH}$ (6-7), these non-glycosylated Cys-rich regions of mucins are conformed with hydrophobic domains hidden in the folds stabilized by the salt bridges between negatively charged carboxylates and positively charged amino groups. At acidic conditions ( $\mathrm{pH} 2$ ), the carboxylates of the salt bridges are protonated what results in breakage of the bridges, unfolding and exposure of hydrophobic regions of protein. In effect, the number of disulphide bonds between cysteine residues is much greater in acidic environment than at neutral $\mathrm{pH}$, and this increases the cross-linking density in the mucus, so its viscosity. High importance of $\mathrm{pH}$ control is also related to the activity of mucolytics such as NAC. The mechanism of its action is related to the thiol-disulfide exchange and it is inhibited at low $\mathrm{pH}$, when the protonated thiol form is more favored than deprotonated thiolate form (Bansil and Turner 2006).

The results are presented in Figs. 4, 5. Viscosity of unmodified mucus samples with higher mucins concentration $(20 \% \mathrm{w} / \mathrm{w})$ measured at physiological value of shear rate $\left(\sim 8 \mathrm{~s}^{-1}\right)$ was $289.4 \pm 7 \mathrm{mPa}$. It corresponds well to the viscosity of the natural mucus in diseased conditions like asthma (146-310 mPas) or cystic fibrosis (app. $160 \mathrm{mPas}$ ) when mucins concentration in bronchial mucus is significantly increased (Rubin 2007). Similarly, the viscosity of samples containing $10 \%$ w/w mucins which equals $16.2 \pm 0.1 \mathrm{mPa}$, is in a good agreement with viscosity of bronchial mucus of healthy subjects (Serisier et al. 2009). 


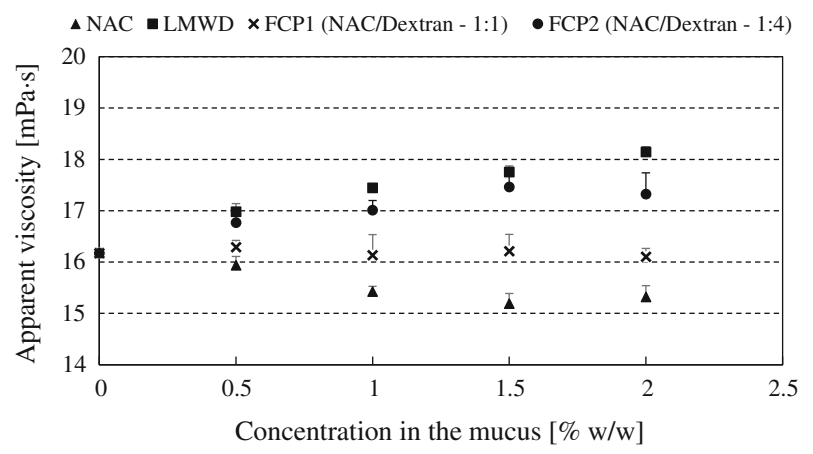

Fig. 5 Comparison of the apparent viscosity of the artificial bronchial mucus modified by functional carrier particles (FCPs) and their components. Average $\pm \mathrm{SD}$, number of replicates $=3$

Based on the data presented in Figs. 4, 5, it can be found that the viscosity of mucus which contains higher contents of mucins is meaningfully reduced in a concentration-dependent manner after addition of pure NAC and FCPs containing NAC. It probably results from gradual breakage of sulphur bonds between mucin molecules. These results also demonstrate that activity of NAC included in the FCPs is preserved after the spray drying process. Higher NAC content always decrease mucus viscosity. It is clearly visible both for samples that contain pure NAC and samples with functional carrier particles containing NAC and dextran in different proportions. The viscosity reduction caused by particles denoted as FCP1 is at average 10-15\% stronger than induced by FCP2. It can be a consequence of a higher content of dextran (LMWD) as the second component of FCPs. The results obtained for pure LMWD added to mucus show that this polysaccharide slightly (by 10-20\%) increases mucus viscosity (Fig. 4). This observation may confirm that mucolytic activity of dextran observed in vivo is mainly related to the osmotic mechanism, which is absent in the studied artificial system.

The highest reduction of viscosity (by $53 \%$ in relation to the blank sample) was obtained for pure NAC at $1.5 \%(\mathrm{w} / \mathrm{w})$ content in the mucus. For $1 \%(\mathrm{w} / \mathrm{w})$ content of FCP1, the original viscosity of the mucus was reduced by $50 \%$. These values should ensure an improvement of the mucociliary clearance rate in the respiratory tract in disease conditions.

For convenient comparison of the obtained results with literature data, the logarithm of the quotient of viscosities: of mucus samples and water, is calculated and listed in Table 3. In all our measurements, the calculated logarithm value is in the range of 2.29-2.40, which closely corresponds to the results reported by Cone et al. and Lai et al., i.e. $2.5 \pm 0.5$ (Cone 2009; Lai et al. 2009). After mucus modification by pure NAC or by both types of functional carrier particles (FCP1, FCP2), the calculated logarithm values decrease to 2.08-2.13 indicating the evident thinning effect.

In the case of mucus model with the lower mucin concentration $(10 \% \mathrm{w} / \mathrm{w})$, the effect of FCPs is not as clear as at the higher concentration (Fig. 5). Particles with equal proportion of LMWD and NAC (FCP1) slightly reduce viscosity, while particles with four times higher content of LMWD in relation to NAC (FCP2) cause increase of mucus viscosity similarly to pure LMWD. These results suggest that mucolytics incorporated into FCPs do not change mucus structure significantly.

The second part of measurements was aimed at determining the effective diffusion coefficient $D_{\text {eff }}$ of Rhodamine B and DSCG in artificial mucus. In vitro methodologies which can be applied for characterization of the molecules mobility in biogels, like mucus, can 
Table 3 Effect of the functional carrier particles, dextran and pure NAC on the logarithm of the quotient of viscosities: of mucus samples with $20 \% \mathrm{w} / \mathrm{w}$ mucin concentration and water (SD- standard deviation, number of repeats: $n=3$ )

\begin{tabular}{lllllllll}
\hline Components & \multicolumn{6}{c}{ Content of examined substances in the mucus $(\% \mathrm{w} / \mathrm{w})$} \\
\cline { 3 - 9 } & & 0 & 0.25 & 0.5 & 0.75 & 1 & 1.5 & 2.0 \\
\hline \multirow{2}{*}{ NAC } & $\log \left(\mu_{\text {mucus }} / \mu_{\mathrm{H}_{2} \mathrm{O}}\right)$ & 2.40 & 2.20 & 2.17 & 2.14 & 2.11 & 2.08 & 2.10 \\
& $\mathrm{SD}(-)$ & 0.03 & 0.17 & 0.19 & 0.13 & 0.02 & 0.01 & 0.15 \\
Dextran (4000Da) & $\log \left(\mu_{\text {mucus }} / \mu_{\mathrm{H}_{2} \mathrm{O}}\right)$ & 2.36 & 2.33 & 2.33 & 2.34 & 2.34 & 2.35 & 2.37 \\
& $\mathrm{SD}(-)$ & 0.04 & 0.08 & 0.04 & 0.05 & 0.06 & 0.05 & 0.15 \\
FCP1 NAC/Dextran 1:1 & $\log \left(\mu_{\text {mucus }} / \mu_{\mathrm{H}_{2} \mathrm{O}}\right)$ & 2.37 & 2.25 & 2.18 & 2.08 & 2.07 & 2.08 & 2.10 \\
& $\mathrm{SD}(-)$ & 0.03 & 0.21 & 0.09 & 0.08 & 0.17 & 0.37 & 0.28 \\
FCP2 NAC/Dextran 1:4 & $\log \left(\mu_{\text {mucus }} / \mu_{\mathrm{H}_{2} \mathrm{O}}\right)$ & 2.36 & 2.29 & 2.17 & 2.15 & 2.11 & 2.13 & 2.16 \\
& $\mathrm{SD}(-)$ & 0.05 & 0.17 & 0.12 & 0.14 & 0.18 & 0.10 & 0.08 \\
\hline
\end{tabular}

be divided into two categories: those measuring the overall drug transport, and the methods that measure the mobility of the drug in local microregions of the biogel. The first group includes the diffusion chamber and semi-infinite slab method (Larhed et al. 1997; Westrin et al. 1994), while the second - fluorescence recovery after photobleaching (FRAP) (Meyvis et al. 1999; Saltzman et al. 1994). Each method has some strong and weak features, however, if information on the drug transfer through the biogel layer to the target (e.g., epithelial cells of the lower airways) is needed, the recommended technique should allow for the measurement of the overall drug transport rate (Sanders et al. 2000). It justifies the methodology applied in this work, i.e., the diffusion chamber approach. One of the most important features of this method is possibility of simulating various disease states or physiological conditions by altering the mucus composition due to addition of selected substances. Simultaneously it should be noted that in in vivo conditions mucus layer is continuously agitated by epithelial cells * cilia. Therefore, the estimation of diffusion coefficient in diffusion cells where the mucus layer is stagnant is only approximation of drug transport in natural conditions. Regardless of this fact, this is still commonly used technique for this kind of measurements.

As the model substances for mass transfer studies were chosen Rhodamine B and antiasthmatic drug-Disodium Cromoglycate (DSCG). The first reason is their molecular mass (479 and 468.3 Da respectively) which is similar to the molecular mass of many drugs used in the treatment of respiratory tract diseases (e.g., budesonide-430.5 Da, fluticasone propionate500.5 Da, formoterol fumarate - 344.4 Da). Secondly, they have properties which significantly simplified the selective and sensitive quantitative determination of transferred material by spectrofluorimetry (Rhodamine B) and spectrophotometry (DSCG).

The composition of analyzed samples and results obtained for rhodamine in mucus reflecting disease conditions are shown in Table 4.

Calculated values of the effective diffusion coefficient $D_{\text {eff }}$ indicate the relationship between the composition and structure of mucus samples, and the mass transfer rate through the mucus layer. The effective diffusion coefficient of Rhodamine B (molecular mass, $M_{\mathrm{r}}=479$ ) calculated for unmodified mucus reflecting disease conditions (mucin concentration: $20 \% \mathrm{w} / \mathrm{w}$ ) is $23.1 \times 10^{-7} \mathrm{~cm}^{2} / \mathrm{s}$ what is approximately 5 times lower than its diffusion coefficient $D_{\text {eff }}$ found in a pure buffer solvent $\left(118.9 \times 10^{-7} \mathrm{~cm}^{2} / \mathrm{s}\right)$. This value is in agreement with the literature data for substances with similar molecular mass: phenolphthalein $\left(M_{\mathrm{r}}=318, D=18 \times 10^{-7} \mathrm{~cm}^{2} / \mathrm{s}\right)$ or phenolphthalein diphosphate 
Table 4 The effective diffusion coefficients $D_{\text {eff }}$ for Rhodamine B in mucus samples reflecting disease ( $20 \%$ w/w mucin concentration), following the addition of mucoactive compounds (SD - standard deviation, number of repeats $-n=3$ )

\begin{tabular}{|c|c|c|c|c|c|}
\hline \multicolumn{2}{|c|}{ Components added to mucus } & \multicolumn{4}{|c|}{ Content of examined substances in the mucus $(\% \mathrm{w} / \mathrm{w})$} \\
\hline & & 0 & 0.5 & 1 & 2.0 \\
\hline \multirow[t]{2}{*}{ NAC } & $\begin{array}{l}\text { Effective diffusion } \\
\text { coefficient } D_{\text {eff }} \text {. } \\
10^{-7}\left(\mathrm{~cm}^{2} / \mathrm{s}\right)\end{array}$ & 23.06 & 31.36 & 47.99 & 50.97 \\
\hline & SD & 3.67 & 3.65 & 5.24 & 5.23 \\
\hline \multirow[t]{2}{*}{ Dextran $(4000 \mathrm{Da})$} & $\begin{array}{l}\text { Effective diffusion } \\
\text { coefficient } D_{\text {eff }} \text {. } \\
10^{-7}\left(\mathrm{~cm}^{2} / \mathrm{s}\right)\end{array}$ & 23.06 & 25.46 & 32.19 & 25.0 \\
\hline & $\mathrm{SD}$ & 3.67 & 2.10 & 4.7 & 6.1 \\
\hline \multirow[t]{2}{*}{ FCP1 NAC/Dextran 1:1 } & $\begin{array}{l}\text { Effective diffusion } \\
\text { coefficient } D_{\text {eff }} \text {. } \\
10^{-7}\left(\mathrm{~cm}^{2} / \mathrm{s}\right)\end{array}$ & 23.06 & 29.50 & 42.31 & 37.02 \\
\hline & SD & 3.67 & 4.93 & 3.21 & 6.22 \\
\hline
\end{tabular}

$\left(M_{\mathrm{r}}=566, D=33 \times 10^{-7} \mathrm{~cm}^{2} / \mathrm{s}\right.$ ), transported through the native porcine mucus (Desai and Vadgama 1991).

Measurements for mucus modified by pure dextran show a slight increase in value of $D_{\text {eff }}$ (up to $32 \times 10^{-7} \mathrm{~cm}^{2} / \mathrm{s}$ ) for dextran concentration in the mucus below $1 \% \mathrm{w} / \mathrm{w}$. Above this concentration, $D_{\text {eff }}$ coefficient decreases which is probably related to the increasing crosslink density in the mucus (by analogy, this effect induces higher viscosity of the mucus). $D_{\text {eff }}$ coefficient for Rhodamine B calculated in mucus with an increasing content of NAC as the main mucolytic ingredient of functional carrier particles, is higher than in the blank sample and equals almost $51 \times 10^{-7} \mathrm{~cm}^{2} / \mathrm{s}$ (at $2 \%$ w/w NAC content). $D_{\text {eff }}$ in the mucus modified by particles FCP1 at $1 \% \mathrm{w} / \mathrm{w}$ concentration, reaches a maximum value of $42.31 \times 10^{-7} \mathrm{~cm}^{2} / \mathrm{s}$. Higher contents of FCP1 in the mucus cause a decrease of $D_{\text {eff }}$ which may be attributed to the presence of dextran incorporated into the FCP (i.e., to increase of viscosity).

The obtained results clearly indicate that the destruction of the mucus structure, which is also related to changes in viscosity, has an influence on the diffusive transport rate of low molecular-weight molecules (Rhodamine B, $M_{\mathrm{r}}=479$ ). However, considering that the average pore size in natural mucus is in the range of 100 to $400 \mathrm{~nm}$ (Cone 2009), it can be expected, that even in case of macromolecular drugs like peptides or proteins with an effective molecular size of 2-15 nm, diffusion should be not drastically retarded (Sanders et al. 2000). Some studies confirm this hypothesis. e.g., work of Saltzman et al. (1994) showed that large macromolecules of human antibodies (IgA: $\left.M_{\mathrm{r}}=150 \mathrm{kDa}, \operatorname{IgM}: M_{\mathrm{r}}=970 \mathrm{kDa}\right)$ readily diffused without restrictions in human cervical mucus $\left(D_{\text {mucus }} / D_{\text {water }}=0.99\right.$ and 0.89 for $\operatorname{IgA}$ and IgM, respectively). Szentkuti (1997) obtained similar results for dextran macromolecules with different molecular masses (10-70 kDa) diffusing in the mucus of distal colon of rats.

On the other hand, Desai and Vadgama (1991) showed that the diffusion of macromolecules like: lysozyme (14.4 kDa), rennet (35 kDa), BSA $(68 \mathrm{kDa})$, and glucose oxidase (186 kDa) through the native gastric porcine mucus was reduced 10 - to 30 -fold in comparison to the diffusion in aqueous solution. Similar observations to ours were also done in the work of Larhed et al. (1997), where two drugs of almost equal molecular weight (approximately two times larger than Rhodamine B), the lipophilic peptide cyclosporin (1204 Da) and the hydrophilic peptide D-arginine vasopressin (1194 Da), diffused with the same rate in pig 
Table 5 The effective diffusion coefficients $D_{\text {eff }}$ for DSCG in mucus samples with $10 \%$ w/w mucin concentration, following the addition of mucoactive compounds (SD - standard deviation, number of repeats $n=3$ )

\begin{tabular}{lc}
\hline Sample composition & $D_{\text {eff }} \cdot 10^{-7} \mathrm{~cm}^{2} / \mathrm{s}( \pm \mathrm{SD})$ \\
\hline Mucin $(10 \% \mathrm{w} / \mathrm{w})$ & $95.2 \pm 2.6$ \\
Mucin $(1 \% \mathrm{w} / \mathrm{w})+$ NAC $(2 \% \mathrm{w} / \mathrm{w})$ & $100.7 \pm 2.2$ \\
Mucin $(1 \% \mathrm{w} / \mathrm{w})+$ LMWD $(2 \% \mathrm{w} / \mathrm{w})$ & $91.7 \pm 1.5$ \\
Mucin $(1 \% \mathrm{w} / \mathrm{w})+$ FCP1 $(2 \% \mathrm{w} / \mathrm{w})$ & $98.7 \pm 1.3$ \\
\hline
\end{tabular}

intestinal mucus, with the rate approximately 6 times slower than in the phosphate buffer. The explanation of these phenomena was mentioned earlier. As a reminder, the presence of impenetrable, slow moving polymer molecules in a solution may lead to an increase in the path length for diffusion. Solute movement may be also retarded by increasing the hydrodynamic drag experienced by the solute as discussed in several papers (Flynn et al. 1974; Muhr and Blanshard 1982; Wesselingh 1993).

Differences between the mentioned above results obtained by several authors can be partly explained by the variety of measurement methods, mucus models and transported molecules used in the separate studies. Probably the largest impact has the fact that the majority of the mentioned studies used the native mucus which could lead to significant differences in the composition of the analyzed mucus samples. It is recognized that the composition has an influence on mucus crosslink density but also on the intensity of other interactions (e.g. ionic, osmotic, hydrophobic) between mucus components and diffusing molecules.

Selection of Rhodamine B as a model drug allows for neglecting the majority of interactions between mucus components and the transferred compound. Due to hydrophilic character, this molecule should not exhibit hydrophobic interactions with mucins and other mucus components (lipids, hydrophobic domains in proteins) (Zhang et al. 2011). Moreover, mucins, are negatively charged due to the presence of $N$-acetylneuraminic acid and sulfated sugars (Carlstedt et al. 1985). Charge of nucleic acids is also negative. Rhodamine B at physiological conditions ( $\mathrm{pH} 7.4$ ) has a neutral charge (it is hold in $\mathrm{pH}$ range 6.0-10.8), therefore it cannot be bounded with the basic mucus components through electrostatic interactions (Garcia et al. 2005; Oh et al. 2008; Schrum et al. 2000).

A large increase of diffusion coefficient after mucus modification by the mucolytic agent also confirms that neither electrostatic nor hydrophobic interactions limit the mass transfer of the drug. It can be concluded that, therefore, the key factor is the crosslink density of the mucus network. The measured influence of pure NAC or FCPs on the drug transport may be slightly overrated, since we used the model with a higher mucin concentration $(20 \%)$ than in natural mucus. Samples with lower mucin content $(10 \% \mathrm{w} / \mathrm{w})$ modified by the addition of NAC, LMWD, and FCP1 (2\% w/w) effective diffusion coefficient were only slightly changed (Table 5).

Simultaneously, these $D_{\text {eff }}$ values were much higher than those obtained for more concentrated mucus, and this leads to correlation between the apparent viscosity of the mucus and the effective diffusion coefficient (Fig. 6).

Finally, it should be noted that in the natural mucus, there are also other ingredients present which are not the target for mucolytic agent. Additionally, the effect caused by mucus modification using FCPs may be not so clear if molecules with different hydrophobic properties and electrostatic charge are transported through the mucus layer. Therefore, further 


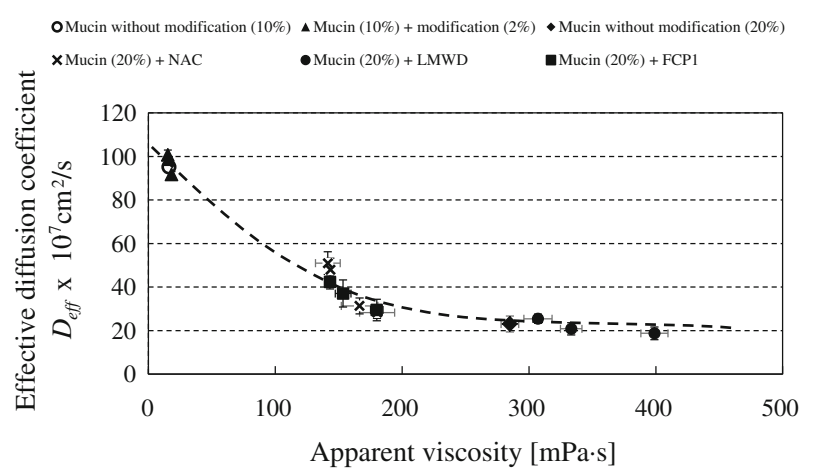

Fig. 6 The value of effective diffusion coefficients as a function of apparent viscosity of analyzed samples

investigations will be needed to characterize more precisely the influence of FCPs on diffusive transport of different drugs in the mucus layer.

\section{Conclusions}

Drug delivered by inhalation may not reach efficiently the target cells due to a low permeability of the mucus which is very viscous during lung disease. According to proposed concept of functional carrier particles (FCPs), we incorporated two mucolytic agents: $N$-acetylcysteine (NAC) and low molecular-weight dextran (LMWD) into solid particles prepared by spraydrying which may be used as drug carriers in aerosol delivery to the lungs using dry powder inhalers (Odziomek et al. 2012). In the current studies, it was demonstrated by in vitro methodology that FCPs indeed may be used to reduce mucus viscosity and increase mucus permeability (i.e., diffusion rate for model drugs). To reflect the properties of natural mucus in different physiological states, the measurements were done for solutions with different concentrations of mucins. The results of this research indicate the relationship between the composition and rheological properties of model mucus, and the mass transport rate. In the case of mucus reflecting diseased conditions, $N$-acetylcysteine, both pure and incorporated into FCP, decreases mucus viscosity, and increases the transport rate of studied drugs. For another FCP component, dextran, which has to be incorporated into FCPs to increase powder stability, only a slight increase of mucus viscosity is observed, and the mass transfer rate is changed very little. At the same time, there was no evident effect in case of mucus with a lower content of mucins (i.e. without disease).

Comparing mucoactivity of the FCPs to the effects measured for their pure individual components, it was possible to demonstrate that FCPs preserve mucolytic activity of the precursor compounds, and that these particles act as enhancers of mass transfer in case of a viscous mucus (lung disease conditions). The obtained results indicate that the concept of FCPs may be useful for pulmonary drug delivery. Obviously, more investigations are needed for a more detailed description of the influence of FCPs on molecular transport through the mucus. Wider representation of drugs with diverse molecular mass, charge, and hydrophobicity should be examined for a better understanding of interactions between mucus, FCPs and the diffusing molecules. The more complete picture of the process may be obtained by incorporating the results of theoretical predictions from molecular dynamics simulations. 
Such studies are currently underway in a wider cooperation (Gniewek and Koliński 2012; Sosnowski et al. 2012).

Acknowledgments This work was financially supported by a grant from National Science Centre based on decision DEC-2011/03/N/ST8/04912. Authors wish to thank Ms. Sylwia Kublik for her help in viscosity measurements. The research was done under umbrella of COST Action MP1106.

Open Access This article is distributed under the terms of the Creative Commons Attribution License which permits any use, distribution, and reproduction in any medium, provided the original author(s) and the source are credited.

\section{References}

Almeida, A.J., Souto, E.: Solid lipid nanoparticles as a drug delivery system for peptides and proteins. Adv. Drug Deliv. Rev. 59(6), 478-490 (2007)

Bansil, R., Turner, B.S.: Mucin structure, aggregation, physiological functions and biomedical applications. Curr. Opin. Colloid Interface Sci. 11, 164-170 (2006)

Bhat, P.G., Flanagan, D.R., Donovan, M.D.: The limiting role of mucus in drug absorption: drug permeation through mucus solution. Int. J. Pharm. 126, 179-187 (1995)

Bhat, P.G., Flanagan, D.R., Donovan, A.D.: Drug binding to gastric mucus glycoproteins. Int. J. Pharm. 134(1-2), 15-25 (1996a)

Bhat, P.G., Flanagan, D.R., Donovan, A.D.: Drug diffusion through cystic fibrotic mucus: steady-state permeation, rheologic properties, and glycoprotein morphology. J. Pharm. Sci. 85(6), 624-630 (1996b)

Brodin, B., Steffansen, B., Nielsen, C.U.: Passive diffusion of drug substances: the concepts of flux and permeability. In: Steffansen, B., Brodin, B., Nielsen, C.U. (eds.) Molecular Biopharmaceutics. Pharmaceutical Press, London (2010)

Carlstedt, I., Sheehan, J.K., Corfield, A.P., Gallagher, J.T.: Mucus glycoproteins: a gel of a problem. Essays Biochem. 20, 40-76 (1985)

Cone, R.A.: Barrier properties of mucus. Adv. Drug Deliv. Rev. 61, 75-85 (2009)

Dawson, M., Krauland, E., Wirtz, D., Hanes, J.: Transport of polymeric nanoparticle gene carriers in gastric mucus. Biotechnol. Prog. 20(3), 851-857 (2004)

Desai, M.A., Vadgama, P.Ł.: Estimation of effective diffusion coefficients of model solutes through gastric mucus: assessment of a diffusion chamber technique based on spectrophotometric analysis. Analyst 116, 1113-1116 (1991)

Feng, W., Speert, D.P., King, M.: Effect of dextran molecular weight on CF sputum rigidity: further evidence for the mechanism of action. Pediatr. Pulmonol. 14S, 273 (1997)

Ferrari, S., Kitson, C., Farley, R., Steel, R., Marriot, C., Parkins, D.A., Scarpa, M., Wainwright, B., Evans, M.J., Coledge, W.H., Geddes, D.M., Alton, M.: Mucus altering agents as adjuncts for nonviral gene transfer to airway epithelium. Gene Ther. 8, 1380-1386 (2001)

Fischer, K.E., Alemán, B.J., Tao, S.L., Hugh, D.R., Li, E.M., Bünger, M.D., Nagaraj, G., Singh, P., Zettl, A., Desai, T.A.: Biomimetic nanowire coatings for next generation adhesive drug delivery systems. Nano Lett. 9(2), 716-720 (2009)

Flynn, G.L., Yalkowsky, S.H., Roseman, T.J.: Mass transport phenomena and models: theoretical concepts. J. Pharm. Sci. 63(4), 479-510 (1974)

Gao, P., Fagerness, P.E.: Diffusion in HPMC gels. I. Determination of drug and water diffusivity by pulsedfield-gradient spin-echo NMR. Pharm. Res. 12(7), 955-964 (1995)

Garcia, A., Ista, L.K., Petsey, D., O‘Brien, M.J., Bisong, P., Mammoli, A.A., Brueck, S.R.J.: Electrokinetic molecular separation in nanoscale fluidic channels. Lab Chip 5, 1270-1276 (2005)

Gniewek, P., Koliński, A.: Coarse-grained modeling of mucus barrier properties. Biophys. J. 102, 195-200 (2012)

Gradoń, L., Marijnissen, J.: Optimization of Aerosol Drug Delivery. Kluwer, Dordrecht (2003)

Heyder, J.: Deposition of inhaled particles in the human respiratory tract and consequences for regional targeting in respiratory drug delivery. Proc. Am. Thorac Soc. 1, 315-320 (2004)

Hirji, M., Franklin, R., Feng, W., Boyd, W.A., King, M.: Effect of oligomeric chain length on mucolytic and mucokinetic effects of oligosacharides. Am. J. Respir. Crit. Care Med. 159, A685 (1998)

King, M., Rubin, B.K.: Pharmalogical approaches to discovery and development of new mucolytic agents. Adv. Drug Deliv. Rev. 54, 1475-1490 (2002) 
King, M.: Mucus and its role in airway clearance and cytoprotection. In: Qutayba, H., Shannon, J., Martin, J. (eds.) Physiologic Basis of Respiratory Disease. BC Decker Inc., Hamilton (2005)

Koushik, K., Kompella, U.B.: Particle and device engineering for inhalation drug delivery. Drug Del. Technol. 4, 40-50 (2004)

Lai, S.K., O’Hanlon, D.E., Harrold, S., Man, S.T., Wang, Y.Y., Cone, R., Hanes, J.: Rapid transport of large polymeric nanoparticles in fresh undiluted human mucus. Proc. Natl. Acad. Sci. USA 104(5), 1482-1487 (2007)

Lai, S.K., Wang, Y.Y., Wirtz, D., Hanes, J.: Micro nad macrorheology of mucus. Adv. Drug Deliv. Rev. 61, 86-100 (2009)

Larhed, A.W., Artursson, P., Grasjo, J., Bjork, E.: Diffusion of drugs in native and purified gastrointestinal mucus. J. Pharm. Sci. 86, 660-665 (1997)

Matsui, H., Wagner, V.E., Hill, D.B., Schwab, U.E., Rogers, T.D., Buttom, B., Taylor, R.M., Superfine, R., Rubinstein, M., Iglewski, R.H., Boucher, R.C.: A physical linkage between cystic fibrosis airway surface dehydration and Pseudomonas aeruginosa biofilms. Proc Natl Acad Sci USA 103, 18131-18136 (2006)

McGill, S.L., Smyth, H.D.: Disruption of the mucus barrier by topically applied exogenous particles. Mol. Pharm. 7(6), 2280-2288 (2010)

Meaney, C., O`Driscoll, C.: Mucus as a barrier to the permeability of hydrophilic and lipophilic compounds in the absence and presence of sodium taurocholate micellar systems using cell culture models. Eur. J. Pharm. Sci. 8(3), 167-175 (1999)

Meyvis, T.L., De Smedt, S.C., Van Oostveldt, P., Demeester, J.: Fluorescence recovery after photobleaching: a versatile tool for mobility and interaction measurements in pharmaceutical research. Pharm. Res. 16, 1153-1162 (1999)

Mortazawi, S.A.: An in vitro assessment of mucus/mucoadhesive interactions. Int. J. Pharm. 124, 173-182 (1995)

Muhr, A.H., Blanshard, J.M.V.: Diffusion in gels. Polymer 23(7), 1012-1026 (1982)

Norris, D.A., Sinko, P.J.: Effect of size, surface charge, and hydrophobicity on the translocation of polystyrene microspheres through gastrointestinal mucin. J Appl. Polym. Sci. 63(11), 1481-1492 (1997)

Odziomek, M., Sosnowski, T.R., Gradoń, L.: Conception, preparation and properties of functional carrier particles for pulmonary drug delivery. Int. J. Pharm. 433, 51-59 (2012)

Oh, Y.J., Gamble, T.C., Leonhardt, D., Chung, C.H., Brueck, S.R., Ivory, C.F., Han, S.M.: Monitoring FET flow control and wall adsorption of charged fluorescent dye molecules in nanochannels integrated into multiple internal reflection infrared waveguied. Lab Chip 8, 251-258 (2008)

Patton, J.S., Brain, J.D., Davies, L.A., Fiegel, J., Gumbleton, M., Kim, K.J., Sakagami, M., Vanbever, R., Erhardt, C.: The particle has landed: characterizing the fate of inhaled pharmaceuticals. J. Aerosol. Med. Pulm. Drug Deliv. 23(2), S71-S87 (2010)

Rubin, B.K.: Mucus structure and properties in cystic fibrosis. Pediatr. Respir. Rev. 8(1), 4-7 (2007)

Saltzman, W.M., Radomsky, M.L., Whaley, K.J., Cone, R.A.: Antibody diffusion in human cervical mucus. Biophys. J. 66, 508-515 (1994)

Sanders, N.N., De Smedt, S.C., Demeester, J.: The physical properties of biogels and their permeability for macromolecular drugs and colloidal drug carriers. J. Pharm. Sci. 89, 835-849 (2000)

Schrum, K.F., Lancaster, J.M., Johnston, S.E., Gilman, S.D.: Monitoring electroosmotic flow by periodic photobleaching of a dilute, neutral fluorophore. Anal. Chem. 72, 4317-4321 (2000)

Serisier, D.J., Carroll, M.P., Shute, J.K., Young, S.A.: Macrorheology of cystic fibrosis, chronic obstructive pulmonary disease \& normal sputum. Respir. Res. 10(63), 1-8, (2009)

Shaw, L.R., Irwin, W.J., Grattan, T.J., Conway, B.R.: The influence of excipients on the diffusion of ibuprofen and paracetamol in gastric mucus. Int. J. Pharm. 290(1-2), 145-154 (2004)

Sosnowski, T.R., Koliński, M., Gradoń, L.: Alteration of surface properties of dipalnitoyl phosphatidylcholine by benzo[a]pyrene: a model of pulmonary effects of diesel exhaust inhalation. J. Biomed. Nanotechnol. 8, 818-825 (2012)

Stern, M., Caplen, N.J., Browning, J.E., Griesenbach, U., Sorgi, F., Huang, L., Gruenert, D.C., Marriot, C., Crystal, R.G., Geddes, M.G., Alton, E.W.: The effect of mucolytic agents on gene transfer across a CF sputum barrier in vitro. Gene Therapy 5, 91-98 (1998)

Sudo, E., Boyd, W.A., King, M.: Effects of dextran sulfate on tracheal mucociliary velocity in dogs. J. Aerosol Med. 13(2), 87-96 (2000)

Szentkuti, L.: Light microscopical observations on luminally administered dyes, dextrans, nanospheres and microspheres in the preepithelial mucus gel layer of the rat distal colon. J. Contr. Release 46, 233-242 (1997)

Truskey, G., Yuan, F., Katz, D.: Transport Phenomena in Biological Systems, 2nd edn. Pearson, Upper Saddle River (2009) 
Varum, F.J.O., Veiga, F., Sousa, J.S., Basit, A.W.: An investigation into the role of mucus thickness on mucoadhesion in the gastrointestinal tract of pig. Eur. J. Pharm. Sci. 40(4), 335-341 (2010)

Wang, Y.Y., Lai, S.K., Suk, J.S., Pace, A., Cone, R., Hanes, J.: Addressing the PEG mucoadhesivity paradox to engineer nanoparticles that "slip" through the human mucus barrier. Angew. Chem. 47(50), 9726-9729 (2008)

Wesselingh, J.A.: Controlling diffusion. J. Control. Release 24(1-3), 47-60 (1993)

Westrin, B.A., Axelsson, A., Zacchi, G.: Diffusion measurement in gels. J. Contr. Release 30, 189-199 (1994)

Yoncheva, K., Lizarraga, E., Irache, J.M.: Pegylated nanoparticles based on poly(methyl vinyl ether-co-maleic anhydride): preparation and evaluation of their bioadhesive properties. Eur. J. Pharm. Sci. 24(5), 411-419 (2005)

Zhang, R., Hummelgadrd, M., Lv, G., Olin, H.: Real time monitoring of the drug release of rhodamine B on graphnene oxide. Carbon 49, 1126-1132 (2011) 